Check for updates

Cite this: Chem. Sci., 2019, 10, 6834

๑ All publication charges for this article have been paid for by the Royal Society of Chemistry

Received 4th March 2019

Accepted 5th June 2019

DOI: $10.1039 / c 9 s c 01068 \mathrm{~h}$

rsc.li/chemical-science

\section{Combinatorial design of multimeric chelating peptoids for selective metal coordination $\dagger$}

\author{
Abel Ricano, $\$^{a}$ Ilya Captain, $\$^{a}$ Korey P. Carter, (D) Bryan P. Nell, (D) ${ }^{a}$ \\ Gauthier J.-P. Deblonde (D a and Rebecca J. Abergel (D) *ab
}

\begin{abstract}
Current methods for metal chelation are generally based on multidentate organic ligands, which are generated through cumbersome multistep synthetic processes that lack flexibility for systematically varying metal-binding motifs. Octadentate ligands incorporating hydroxypyridinone or catecholamide binding moieties onto a spermine scaffold are known to display some of the highest affinities towards $f$ elements. Enhancing binding affinity for specific lanthanide or actinide ions however, necessitates ligand architectures that allow for modular and high throughput synthesis. Here we introduce a highthroughput combinatorial library of 16 tetrameric $N$-substituted glycine oligomers (peptoids) containing hydroxypyridinone or catecholamide chelating units linked via an ethylenediamine bridge and, for comparison, we also synthesized the corresponding mixed ligands derived from the spermine scaffold: 3,4,3-LI(1,2-HOPO $)_{2}(\mathrm{CAM})_{2}$ and 3,4,3-LI(CAM) 2 (1,2-HOPO) $)_{2}$. Coordination-based luminescence studies were carried out with $\mathrm{Eu}^{3+}$ and $\mathrm{Tb}^{3+}$ to begin probing the properties of the new ligand architecture and revealed higher sensitization efficiency with the spermine scaffold as well as different spectroscopic features among the structural peptoid isomers. Solution thermodynamic properties of selected ligands revealed different coordination properties between the spermine and peptoid analogues with $\mathrm{Eu}^{3+}$ stability constants $\log \beta_{110}$ ranging from $28.88 \pm 3.45$ to $43.97 \pm 0.49$. The general synthetic strategy presented here paves the way for precision design of new specific and versatile ligands, with a variety of applications tailored towards the use of f-elements, including separations, optical device optimization, and pharmaceutical development.
\end{abstract}

\section{Introduction}

Interest in f-block elements has increased substantially in recent years as these elements have been used in a growing number of applications such as luminescent phosphors ${ }^{\mathbf{1}, 2}$ and diagnostic imaging and therapy. ${ }^{3-6}$ While the radioactive $5 \mathrm{f}$ actinides (An) have garnered significant attention due to the legacy of their use in energy and weapons, ${ }^{7-11}$ lanthanides (Ln), aside from promethium (Pm), consist wholly of stable isotopes. Many of the applications that utilize Ln metals are based on transitions within the $4 \mathrm{f}$ orbitals, which are shielded from external perturbations by filled $5 \mathrm{~s}$ and $5 \mathrm{p}$ orbitals. This means that the $4 \mathrm{f}$ orbitals of Ln metal centers do not appreciably

\footnotetext{
${ }^{a}$ Chemical Sciences Division, Lawrence Berkeley National Laboratory, Berkeley, CA 94720, USA. E-mail: abergel@berkeley.edu

${ }^{b}$ Department of Nuclear Engineering, University of California, Berkeley, CA 94720, USA $\dagger$ Electronic supplementary information (ESI) available: Detailed experimental procedures, LC-MS, high resolution MS, TOF-MS/MS, and NMR spectra for peptoid ligands, UV-vis and luminescence spectra for Eu and Tb peptoid and 343-LI complexes, luminescence lifetime values, example data sets from solution thermodynamic titrations, and quantum yield data are provided. See DOI: $10.1039 / \mathrm{c} 9 \mathrm{sc} 01068 \mathrm{~h}$

\$ Equal contributions.
}

participate in bonding, thus local coordination environments do not overtly influence their respective solution or solid-state electronic and photophysical properties.

Harnessing the unique capabilities of lanthanide metal cations so that one may selective tune the resulting properties involves exercising control over the first coordination sphere of Ln(III) ions. Using high-affinity, chelating ligands is one pathway for controlling Ln local coordination geometry, and currently the highest affinity f-block binding is achieved by organic, bio-inspired ligands. ${ }^{\mathbf{1 2 - 1 4}}$ Raymond and coworkers ${ }^{\mathbf{1 5}}$ have demonstrated the most striking example of f-block metal cation specificity by preparing a macrocyclic ligand that chelates $\mathrm{Th}^{4+}$ with a formation constant $\left(K_{\mathrm{f}}\right)$ of $10^{54}$, yet similar examples are limited as synthesis of such molecular structures is time consuming, expensive, and often not modular. As a result, new synthetic methods are needed to develop ligands in a high-throughput manner, which can also provide improved understanding of the subtleties of 4 f-element coordination chemistry.

Most ligands with high-affinity for the f-block are multidentate and are composed of small metal-binding moieties placed on a molecular scaffold. ${ }^{7}$ Spermine (also called 3,4,3-LI) is described as a privileged scaffold due to the large number of 
ligands that have been derived from it and their relatively facile, low-cost, synthesis. Ligands of form X (Fig. 1) are typically synthesized by reacting spermine with activated esters of bidentate moieties and often display impressive binding characteristics. Mixed ligands of form Y (Fig. 1) that contain two different types of binding groups can be prepared by exploiting reactivity differences between the 3,4,3-LI primary and secondary amines, but are not very common due to low synthetic yields. Further variation of binding units along the 3,4,3-LI backbone has not been done due to impractical and low yielding syntheses of form $\mathrm{Z}$ ligands (Fig. 1). Access to ligands of the latter type is important, as it would allow for systematic binding unit variation along the backbone, leading to an enhanced understanding of Ln-ligand interactions.

To circumvent synthetic hurdles in the 3,4,3-LI system, we developed a new strategy by incorporating high-affinity metalbinding units onto peptoid scaffolds. Peptoids are structural analogs of peptides composed of $\mathrm{N}$-substituted glycine oligomers. They are composed of primary amine sub-monomers, can be synthesized on solid support, and may be prepared using traditional automated peptide synthesizers. ${ }^{16}$ The combinatorial nature of peptoid synthesis and asymmetric scaffold provides a unique opportunity to control binding moiety sequence. This method can yield thousands of ligands in a matter of days, yet most studies have been limited to using commercially available amine sub-monomers. Peptoids have been examined as potential binders for a number of metals using combinatorial libraries, ${ }^{17-22}$ and here we aimed to improve upon the process of peptoid ligand synthesis by using custom-made sub-monomers known for their f-element binding properties. In this pilot study, we incorporated two well-characterized binding moieties into the peptoid scaffold using standard synthetic methods. The modularity of the scaffold allowed for the rapid preparation of all 16 possible tetrameric peptoid, octadentate ligands. In parallel, we also prepared two spermine-based ligands of form Y (Fig. 1) to serve as baseline comparisons. We then carried out luminescencebased coordination studies on all resulting ligands using $\mathrm{Eu}^{3+}$ and $\mathrm{Tb}^{3+}$, and stability constants for selected chelators with $\mathrm{Eu}^{3+}$ were determined. These systematic studies revealed that slight variations in ligand structure could result in significant differences in spectroscopic properties and complex stability, even for chemically similar Ln elements.

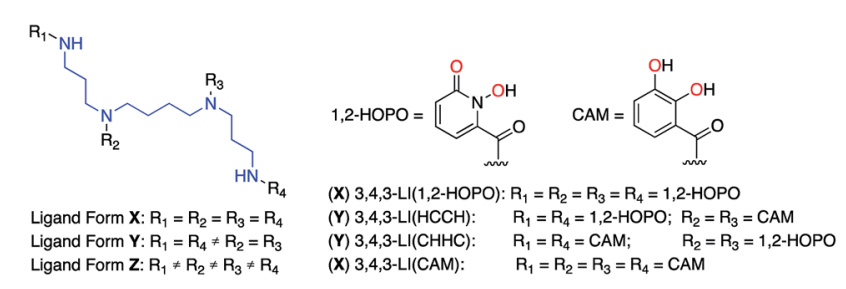

Fig. 1 Spermine-based octadentate ligands. The 3,4,3-LI scaffold (blue) is connected to four metal-binding units $\left(R_{1}, R_{2}, R_{3}\right.$, and $\left.R_{4}\right)$. With 1,2-HOPO and CAM (metal-binding atoms highlighted in red), only four ligand combinations are easily accessible through classical synthetic methods: 3,4,3-LI(1,2-HOPO), 3,4,3-LI(HCCH), 3,4,3$\mathrm{LI}(\mathrm{CHHC})$, and 3,4,3-LI(CAM).

\section{Results and discussion}

\section{Synthesis of peptoid library}

Bidentate metal-binding units 1-hydroxy-pyridin-2-one (1,2HOPO) and catecholamide (CAM) are well known moieties in felement binding (Fig. 1). ${ }^{7}$ Extensive studies have established the octadentate 3,4,3-LI(1,2-HOPO) as a flagship chelator for An and Ln decorporation, ${ }^{13,23}$ and 3,4,3-LI(CAM) was recently evaluated for $\mathrm{Eu}^{3+}, \mathrm{Th}^{4+}$, and $\mathrm{Zr}^{4+}$ binding (Fig. 1). ${ }^{14}$ To accommodate the larger ionic radii of f-elements upon chelation, ethylenediamine bridges were inserted within the sub-monomer units to link 1,2HOPO and CAM moieties to the peptoid scaffold (Scheme 1). Primary amine sub-monomers that we term $\mathbf{H}$ and $\mathbf{C}$ (corresponding to ethylenediamine-substituted 1,2-HOPO and CAM, respectively) were synthesized by reacting known precursor acyl chlorides with excess ethylenediamine to ensure single addition of chelating moieties (Scheme 1). The benzyl group was used to protect the $N$-hydroxyl of $\mathbf{H}$ while a diphenylacetal was used for the 1,2-diol on the $\mathbf{C}$ sub-monomer, and both sub-monomers were characterized by ${ }^{1} \mathrm{H}$ and ${ }^{13} \mathrm{C}$ NMR spectroscopy (ESI $\dagger$ ). Acid labile protecting groups were chosen so they would be removed during cleavage of peptoids from solid support using trifluoroacetic acid (TFA).

$\mathbf{H}$ and $\mathbf{C}$ sub-monomers were then used to prepare the peptoids on Rink Amide resin employing standard coupling chemistry. In lieu of automated peptoids synthesis, we used fritted syringes as reaction vessels, which allowed for recovery of unreacted sub-monomers for future use. We were able to successfully reuse the solution of sub-monomer up to three times without any decrease in performance. Spent solutions were concentrated and re-purified by column chromatography.

Subsequent test cleavages on small samples of resin revealed that benzyl groups were not completely removed from $\mathbf{H}$ sub-

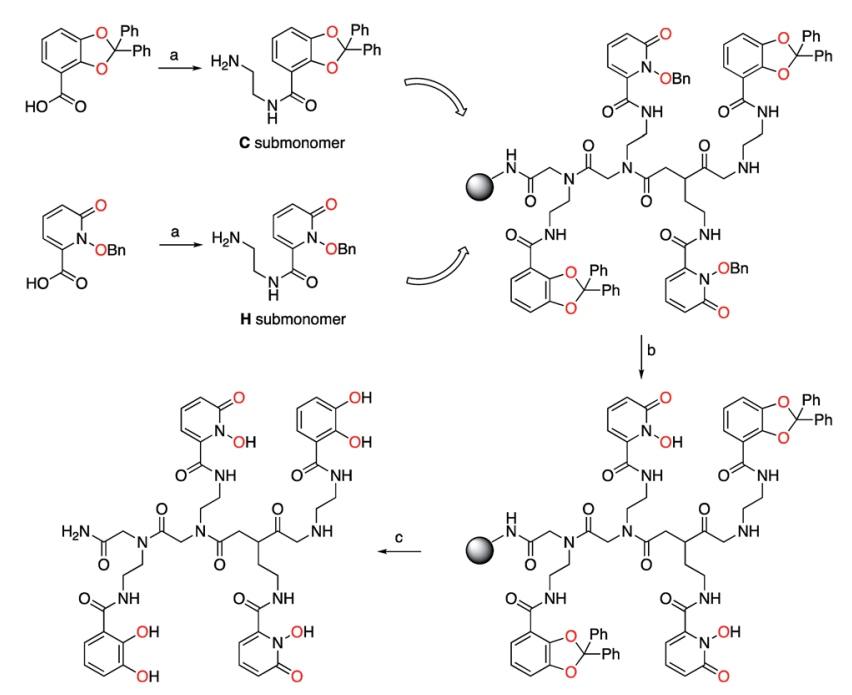

Scheme 1 Synthesis of tetrameric peptoid ligands. (a) Oxalyl chloride, then ethylenediamine. (b) $0.1 \mathrm{M} \mathrm{BBr}_{3}$ in $\mathrm{CH}_{2} \mathrm{Cl}_{2}$. (c) 95/2.5/2.5 - TFA/ water/triisopropylsilane. One peptoid structure, $\mathrm{CHHC}$, shown, out of 16 possible tetrameric combinations with the two different types of sub-monomers $\mathrm{C}$ and $\mathrm{H}$. Metal-binding oxygens are highlighted in red. 
monomers during TFA treatment, even with prolonged reaction times. After screening a number of conditions, we found that addition of $5 \%$ concentrated aqueous $\mathrm{HCl}$ to the TFA cleavage cocktail afforded the deprotected product but also led to significant peptoid backbone cleavage, which greatly complicated purification. Post-cleavage hydrogenolysis yielded the desired products but was impractical on milligram scales at which the peptoids were prepared. Alternatively, we found that

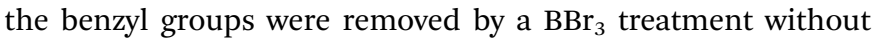
cleaving the peptoid from solid support. This allowed us to deprotect the peptoids and easily remove byproducts using a solvent wash. We finally settled on treating the resin with $0.1 \mathrm{M} \mathrm{BBr}_{3}$ in dichloromethane $\left(\mathrm{CH}_{2} \mathrm{Cl}_{2}\right)$ followed by TFA cleavage to obtain the deprotected crude peptoid ligands. The ligands were purified using reverse phase preparative HPLC and were characterized by ${ }^{1} \mathrm{H}$ and ${ }^{13} \mathrm{C}$ NMR spectroscopy as well as high-resolution mass spectrometry, with TOF-MS/MS used to verify the peptoid sequence (ESI, Fig. S1-S23†). Ligands containing $\mathbf{H}$ moieties were generally more difficult to work with and to purify due to their propensity to bind iron and other trace metals present in instrument components even in acidic media. Due to the asymmetric nature of the peptoid scaffold (Scheme 1), the formation of $2^{n}$ ligands is possible, where 2 denotes the number of sub-monomers ( $\mathbf{H}$ and $\mathbf{C})$ used and " $n$ " the oligomer length. Sufficient amounts of all 16 possible tetrameric peptoid ligands were obtained in pure form. The synthesized peptoid chelators are listed in Table 1.

\section{Synthesis of 3,4,3-LI mixed ligands}

In order to compare our new peptoid scaffold to the 3,4,3-LI backbone, we prepared two mixed derivatives, each either bearing two 1,2-HOPO units on the two internal tertiary amines and two CAM subunits on the terminal secondary amines of the spermine backbone $\left(3,4,3-\mathrm{LI}(\mathrm{CAM})_{2}(1,2-\mathrm{HOPO})_{2}\right.$, adopting the same binding group sequence as peptoid CHHC), or vice versa $\left(3,4,3-\mathrm{LI}(1,2-\mathrm{HOPO})_{2}(\mathrm{CAM})_{2}\right.$, adopting the same binding group sequence as peptoid $\mathbf{H C C H}$ ). This synthesis takes advantage of the higher reactivity of the primary amines of spermine. One of the acid derivatives of 1,2-HOPO or CAM (with hydroxyl functionalities protected by benzyl or methyl groups, respectively) is first added onto the terminal amines through reaction with spermine in a controlled, dilute manner, and in the presence of amide coupling reagent carbonyldiimidazole (CDI). The acid chloride of the other subunit is then made in situ and directly added to the internal amines. Both methyl and benzyl protecting groups could be removed by $\mathrm{BBr}_{3}$ treatment, providing the

Table 1 Synthesized Peptoid Library ${ }^{a}$

$\begin{array}{llll}\text { (1) } \mathrm{HHHC} & (2) \mathrm{CHHH} & (3) \mathrm{HCHH} & (4) \mathrm{HHCH} \\ \text { (5) CHHC } & \text { (6) HHCC } & \text { (7) CCHH } & \text { (8) HCHC } \\ \text { (9) HCCH } & \text { (10) CHCH } & \text { (11) HCCC } & \text { (12) CHCC } \\ \text { (13) CCHC } & \text { (14) CCCH } & \text { (15) CCCC } & \text { (16) HHHH }\end{array}$

${ }^{a}$ Synthesized tetrameric peptoids with $\mathbf{H}$ and $\mathbf{C}$ binding moieties. The leftmost designation in the ligand name denotes the first added moiety on the peptoid scaffold (i.e. nearest the terminal amide). crude ligands, which were purified using reverse phase preparative HPLC and characterized by ${ }^{1} \mathrm{H}$ and ${ }^{13} \mathrm{C}$ NMR spectroscopy and high-resolution mass spectrometry (ESI $\dagger$ ).

\section{UV-visible spectroscopic properties}

The electronic absorption spectra (ESI, Fig. S24 $\dagger$ ) of all sixteen peptoid ligands revealed the characteristic $\pi-\pi *$ transitions of each aromatic system. (16) HНHн $\left(\lambda_{\max }=348 \mathrm{~nm}\right)$ and (15) CCCC $\left(\lambda_{\max }=320 \mathrm{~nm}\right)$ served as upper and lower bounds, respectively, for the maximum absorption of the peptoid ligands. Absorption maxima of peptoid ligands with more $\mathbf{H}$ units were red-shifted when compared with the multi-C unit peptoids (Fig. 2A), but featured similar extinction coefficients (Fig. 2B). Both 3,4,3-LI(CAM) and 3,4,3-LI(HCCH) free ligands contained a small shoulder around $285 \mathrm{~nm}$ in addition to their main absorption band centered at $320 \mathrm{~nm}$ and $350 \mathrm{~nm}$, respectively. The absorption band for free 3,4,3- $\mathrm{LI}(\mathrm{CHHC})$ was centered at approximately $322 \mathrm{~nm}$ and changed in intensity and width upon complexation. All the complexes (of both $\mathrm{Eu}^{3+}$ and $\mathrm{Tb}^{3+}$ ) exhibited similar absorbance maxima at $c a .330 \mathrm{~nm}$, with
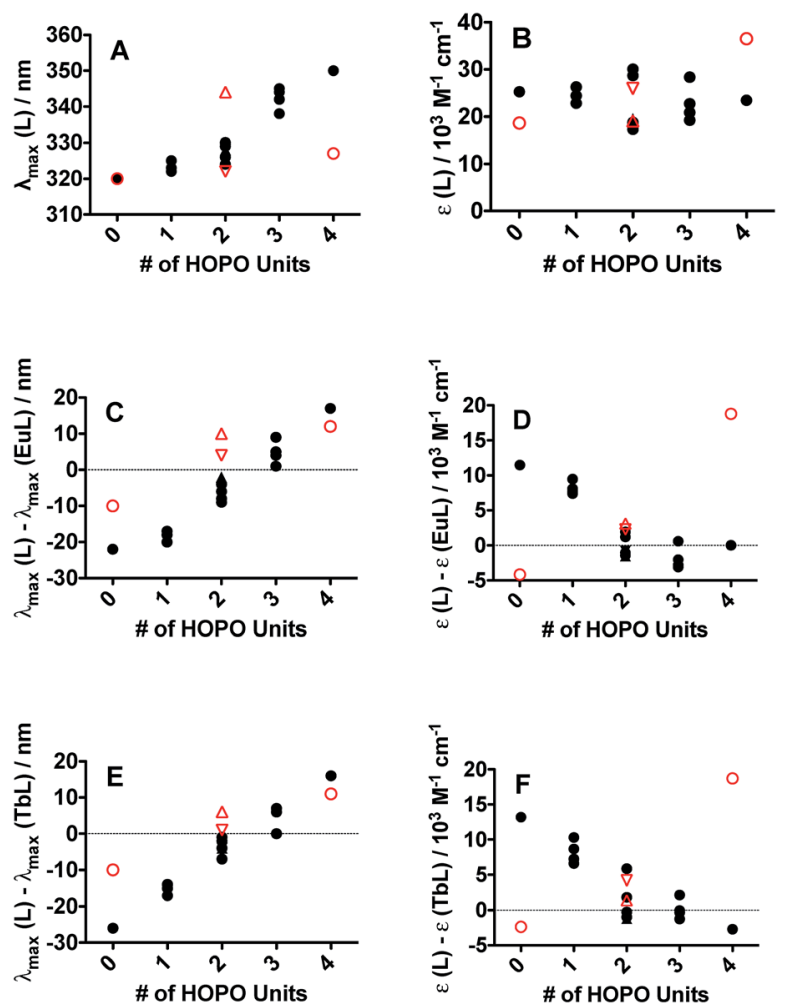

Fig. 2 Ligand and complex UV-vis spectroscopic parameters dependence as a function of the number of 1,2-HOPO metal-binding units (0 for CCCC and 3,4,3-LI(CAM) to 4 for HHHH and 3,4,3-LI(1,2HOPO)) on the peptoid (black full circles) and spermine-based scaffolds (red open symbols; circles for 3,4,3-LI(CAM) and 3,4,3-LI(1,2$\mathrm{HOPO})$; upward and downward triangles for 3,4,3- $\mathrm{LI}(\mathrm{HCCH})$ and 3,4,3-LI(CHHC), respectively). (A) Ligand maximum absorption wavelength. (B) Ligand extinction coefficient. (C) Wavelength shift upon Eu complexation. (D) Change in extinction coefficient upon Eu complexation. (E) Wavelength shift upon Tb complexation. (F) Change in extinction coefficient upon Tb complexation. 
Ln-peptoid complexes with multiple $\mathbf{H}$ units blue-shifted with respect to the free ligands and vice versa for those with multiple C moieties (Fig. 2C and $\mathrm{E}$ for $\mathrm{Eu}^{3+}$ and $\mathrm{Tb}^{3+}$ complexes, respectively). Extinction coefficients decreased significantly upon $\mathrm{Eu}$ or $\mathrm{Tb}$ complexation for peptoid ligands containing more than $2 \mathbf{C}$ sub-monomers, whereas the opposite trend was observed for the spermine-based ligands (Fig. 2D and $\mathrm{F}$ for $\mathrm{Eu}^{3+}$ and $\mathrm{Tb}^{3+}$ complexes, respectively).

\section{Ligand-sensitization of $\mathrm{Eu}^{3+}$ and $\mathrm{Tb}^{3+}$ luminescence}

The ligand of form $\mathrm{X}$ (Fig. 1) that bears 1,2-HOPO units (i.e. $3,4,3-\mathrm{LI}(1,2-\mathrm{HOPO}))$ is known to sensitize the luminescence emission of selected $\mathrm{Ln}$ (III) cations through the formation of stable stoichiometric complexes and the so-called "antenna effect", ${ }^{23,24}$ as depicted through a simplified Jablonski diagram in Fig. 3. To this end, we sought to determine if the peptoid ligands could also sensitize Ln(III) ions in a similar fashion, using $\mathrm{Eu}^{3+}$ and $\mathrm{Tb}^{3+}$ as representative systems. The emission spectra of the $\mathrm{Eu}^{3+}$ and $\mathrm{Tb}^{3+}$ peptoid complexes revealed relatively weak signals (ESI, Fig. S25-S30 $)$ ). Quantum yields of Eu:HHCH (4) $(\phi=0.0043 \pm 0.0002)$ and Tb:3,4,3-LI(CHHC) $(\phi=$ $0.0011 \pm 0.0001)$ were determined by the optical dilution method. These values were then used as benchmarks to estimate the values for the remaining complexes (ESI, Table S1 $\dagger$ ) by taking the ratio of the integrated emission spectra to that of the corresponding metal complex, assuming identical extinction coefficients. Here we can use Eu:3,4,3-LI(1,2-HOPO) as a reference with an estimated value of 0.14 , consistent with the published quantum yield values of $15.6 \pm 0.6 \%$ (isolated complex) and $14.0 \pm 0.3 \%$ (complex formed in situ). ${ }^{25}$ This comparative method is not meant to be interpreted as rigorous for quantum yield determination, rather we aimed to probe relevant variations within the large number of ligands and the small scale of the pilot study. The largest quantum yields for $\mathrm{Eu}^{3+}$ and $\mathrm{Tb}^{3+}$, not including 3,4,3-LI(1,2-HOPO), are complexes formed with (16) $\mathbf{H H H H}$ and $3,4,3-\mathrm{LI}(\mathrm{CHHC})$, respectively. Nonetheless,

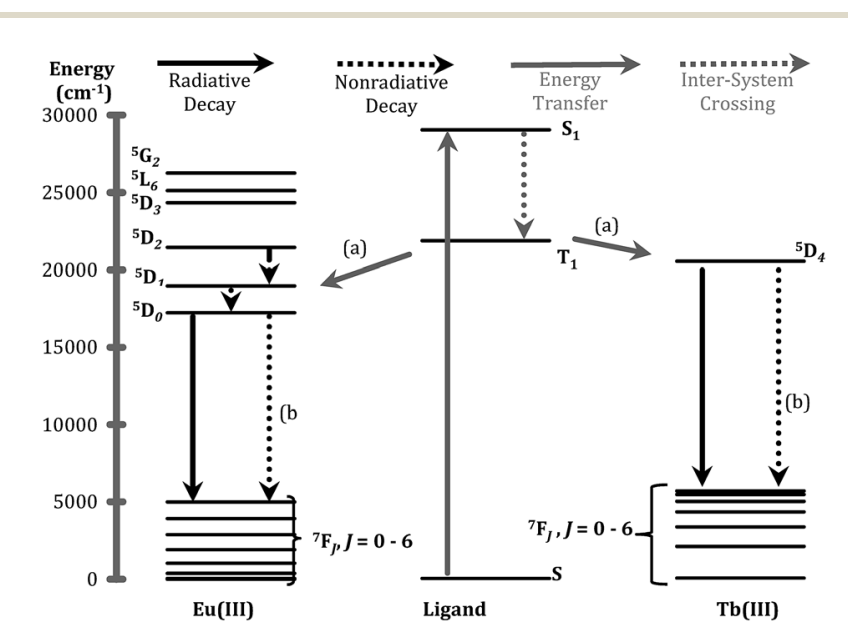

Fig. 3 Jablonski diagram representing the major energy transfer pathways of sensitization. (a) Represents energy transfer from the ligand to the metal; (b) indicates non-radiative de-excitation. Energy levels for free ions were adapted from previous publications. ${ }^{26,27}$ these observed weak emission properties provide evidence of poor energy transfer to the metal ([a] in Fig. 3), significant nonradiative de-excitation ([b] in Fig. 3), or a combination thereof.

One of the critical components in $\operatorname{Ln}$ (III) sensitization is the energy transfer efficiency between the first triplet-state of the ligand $\left(\mathrm{T}_{1}\right)$ and the accepting state of the metal (Fig. 3$) .{ }^{28}$ Since $\mathrm{Gd}^{3+}$ exhibits a size and atomic weight similar to $\mathrm{Eu}^{3+}$ but lacks an appropriately positioned electronic acceptor level, the tripletstate of the ligand can be estimated by measuring the phosphorescence spectra of the respective Gd(III) complex. Energy transfer is most efficient when the respective resonance levels are similar enough in energy such that thermal back-transfer can be minimized. For $\mathrm{Eu}^{3+}$ and $\mathrm{Tb}^{3+}$, this occurs when the triplet-state donor level is 2500-3500 $\mathrm{cm}^{-1}$ and 2500$4000 \mathrm{~cm}^{-1}$, respectively, higher than the metal accepting state. ${ }^{29}$ Triplet state energies were determined for all investigated compounds, according to described methods. ${ }^{30,31}$ The $\mathrm{T}_{1}$ energies were found close to the $\mathrm{Eu}^{3+5} \mathrm{D}_{2}$ level, similar to what has been reported with polyaminocarboxylates ${ }^{28}$ but contrasting with ligands such as Schiff bases ${ }^{32}\left({ }^{5} D_{0}\right)$ or $\beta$-diketonates ${ }^{33}\left({ }^{5} D_{1}\right)$. Structures with triplet state energies values nearest to the ${ }^{5} \mathrm{D}_{2}$ level resulted, overall, in higher $\mathrm{Eu}$ emission intensities (Fig. 4A). To further probe the role of each HOPO or CAM binding unit on luminescence sensitization, phosphorescence spectral shapes were examined in more details for each Gd complex. Deconvolution of the main triplet-state peak of (15) CCCC and (16) HHHH with two Gaussian curves each revealed
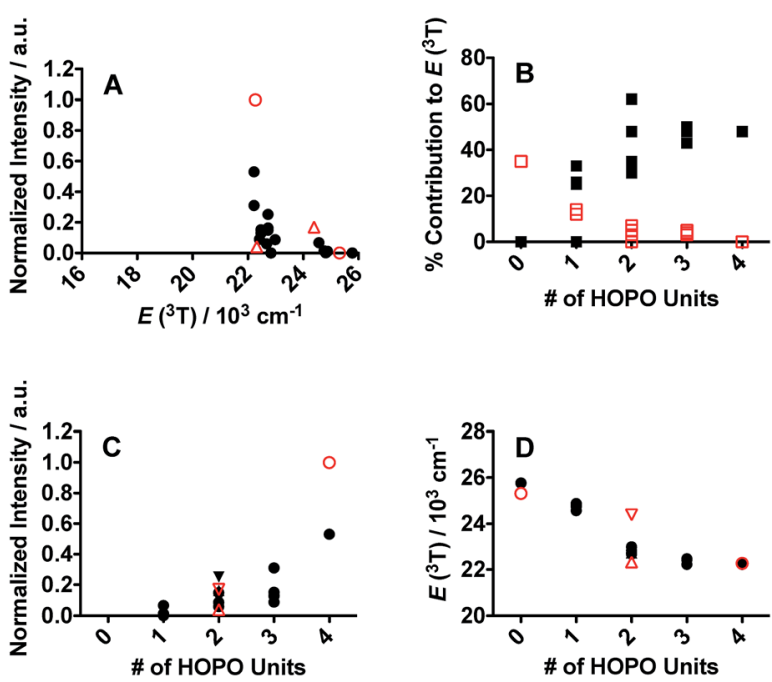

Fig. 4 (A) Eu luminescence emission intensity as a function of ligand triplet-state energy for the peptoid (black full circles) and sperminebased scaffolds (red open symbols; circles for 3,4,3-LI(CAM) and 3,4,3$\mathrm{LI}(1,2-\mathrm{HOPO})$; upward and downward triangles for 3,4,3- $\mathrm{LI}(\mathrm{HCCH})$ and 3,4,3- $\mathrm{LI}(\mathrm{CHHC})$, respectively). (B) \% contribution of the 1,2-HOPO (full black squares) and CAM (open red squares) metal-binding units to the phosphorescence from the triplet excited state as a function of the number of 1,2-HOPO metal-binding units for peptoid ligands. (C) Eu luminescence emission intensity and (D) ligand triplet-state energy as a function of the number of 1,2-HOPO metal-binding units ( 0 for CCCC and 3,4,3-LI(CAM) to 4 for HHHH and 3,4,3-LI(1,2-HOPO)) on the peptoid and spermine-based scaffolds; symbols are as described for panel A. 
bands centered around $20000 \mathrm{~cm}^{-1}(\sim 498 \mathrm{~nm})$ for both, $18100 \mathrm{~cm}^{-1}(\sim 552 \mathrm{~nm})$ for (16) HHHH, and a higher energy contribution from (15) CCCC centered around $21400 \mathrm{~cm}^{-1}$ ( $\sim 467 \mathrm{~nm})$. There was an additional small band in the (16) НHНH phosphorescence spectra centered around $25000 \mathrm{~cm}^{-1}$ $(\sim 395 \mathrm{~nm})$ that was also observed for (5) CHHC $(\sim 391 \mathrm{~nm})$. The extent of 1,2-HOPO and CAM triplet-state character in the rest of the peptoid ligands was gauged by the relative contribution of the spectral bands centered at $18100 \mathrm{~cm}^{-1}$ and $21400 \mathrm{~cm}^{-1}$, respectively, since both (15) CCCC and (16) HHHH featured a peak centered at $c a .20000 \mathrm{~cm}^{-1}$ (Fig. 4B). Luminescence intensity was found to increase with the number of $\mathbf{H}$ moieties on the peptoid scaffold (Fig. 4, panels C and D, and ESI $\dagger$ ), which was notable as the 1,2-HOPO triplet-state contribution is just under $1000 \mathrm{~cm}^{-1}$ above the ${ }^{5} \mathrm{D}_{2}$ manifold of $\mathrm{Eu}(\mathrm{III})$, much lower than the $2500 \mathrm{~cm}^{-1}$ energy gap suggested by Latva et al. ${ }^{28}$ for optimal energy transfer.

The triplet-state data of the spermine based ligands was similarly treated (Fig. 4). 3,4,3-LI(CAM) and 3,4,3-LI(1,2HOPO) had deconvoluted bands centered around $22000 \mathrm{~cm}^{-1}(\sim 455 \mathrm{~nm})$ and $18200 \mathrm{~cm}^{-1}$ ( $\left.\sim 50 \mathrm{~nm}\right)$, respectively, and an additional component around $19800 \mathrm{~cm}^{-1}$ $(\sim 506 \mathrm{~nm})$. As in the peptoid analogue, 3,4,3-LI(1,2-HOPO) had an additional small peak centered around $24400 \mathrm{~cm}^{-1}$ ( $\sim 410 \mathrm{~nm})$. A larger triplet-state character from 3,4,3-LI(CAM) was observed in 3,4,3- $\mathrm{LI}(\mathrm{CHHC})$ than in the 3,4,3- $\mathrm{LI}(\mathrm{HCCH})$. As with the peptoid scaffold ((15) CCCC), no fluorescence was observed for 3,4,3-LI(CAM) complexed with $\mathrm{Eu}^{3+}$. There was about a $\sim 30 \%$ weaker quantum yield for the Eu complex with 3,4,3-LI(HCCH) than 3,4,3-LI(CHHC), but this could very well be attributed to the low molar absorptivity (vide infra) of the 3,4,3-LI(HCCH) complex.

Further analysis of the Eu emission spectra revealed Stark splitting and small shifts in the ${ }^{5} \mathrm{D}_{0} \rightarrow{ }^{7} \mathrm{~F}_{2}$ transition for most complexes (ESI, Fig. S25-S30†). Two comparisons are presented in Fig. 5. A $2 \mathrm{~nm}$ shift between the peaks of the Eu:1,2-HOPO complexes, 3,4,3-LI(1,2-HOPO) and (16) $\mathbf{H H H H}$, as well as a shoulder peak at $618.5 \mathrm{~nm}$ for Eu:3,4,3-LI(1,2-HOPO) was observed (Fig. 5A). Panel B of Fig. 5 shows the directional isomers (3) HCHH and (4) HHCH and highlights the substantial differences that can result from small changes in the placement of $\mathbf{H}$ and $\mathbf{C}$ binding moieties. We note an approximate 4 -fold intensity increase when comparing the Eu complexes of $\mathbf{3}$ and $\mathbf{4}$,
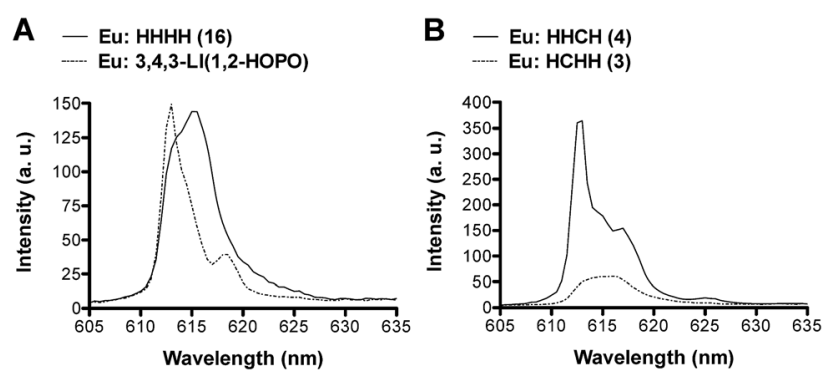

Fig. 5 The ${ }^{5} D_{0} \rightarrow{ }^{7} F_{2}$ emissive transition of the Eu complexes formed with (A) 3,4,3-LI(1,2-HOPO) (dotted line) and (16) $\mathrm{HHHH}$ (solid line); (B) (3) $\mathrm{HCHH}$ (dotted line) and (4) $\mathrm{HHCH}$ (solid line). along with some Stark splitting of the ${ }^{7} \mathrm{~F}_{2}$ transition for 4 that is not observed with the analogous Eu:HCHH (3) complex.

The $\mathrm{Tb}^{3+}$ spectra revealed much less efficient sensitization than with $\mathrm{Eu}^{3+}$ congeners as all spectra had significant ligand luminescence leakage around $450 \mathrm{~nm}$. None of the triplet-states for any of the investigated ligands had optimum energy to efficiently populate the $\mathrm{Tb}^{3+}$ accepting level $\left(\sim 20500 \mathrm{~cm}^{-1}\right)$ and there was a significant difference in the sensitization efficiencies between the $\mathrm{Eu}(\mathrm{III})$ and $\mathrm{Tb}(\mathrm{III})$ systems. For $\mathrm{Eu}^{3+}$ the most luminescent peptoid was (16) НHНH, followed by (4) НHCH, whereas with $\mathrm{Tb}^{3+}$ it was (2) СНHН.

Luminescence lifetimes of Eu:peptoid complexes as well as 3,4,3-LI mixed ligand complexes with $\mathrm{Eu}^{3+}$ and $\mathrm{Tb}^{3+}$ revealed biexponential decays with the exception of Eu:HHCH (4), Eu:CHHC (5), both mono-exponential decays, and Eu:HCCC (11) and Tb:3,4,3-LI(CHHC), which were fit as tri-exponential. The longest lifetimes came from Eu complexes of (16) $\mathbf{H H H H}$ $(827 \mu \mathrm{s})$ and $3,4,3-\mathrm{LI}(\mathrm{CHHC})(733 \mu \mathrm{s})$, both corresponding to one coordinated water molecule according to Kimura's empirical equation (ESI, Table S2 †). ${ }^{34}$ Comparing (16) HHHH $(827 \mu \mathrm{s})$ with $3,4,3-\mathrm{LI}(1,2-\mathrm{HOPO})(805 \mu \mathrm{s}),{ }^{24}$ reveals that when all binding units are 1,2-HOPO moieties there is little variation between 3,4,3-LI and peptoid scaffolds. With peptoid ((5) CHHC and (9) HCCH) and 3,4,3-LI mixed ligands each featuring two $\mathbf{C}$ and two $\mathbf{H}$ units the results get more interesting as we note a small (64 $\mu \mathrm{s}$ ) increase in $\mathrm{Eu}(\mathrm{III})$ lifetime when going from (9) to 3,4,3$\mathrm{LI}(\mathrm{HCCH})$ and a more substantial change $(180 \mu \mathrm{s})$ when going from (5) to 3,4,3-LI(CHHC) (ESI, Table S2 $\dagger$ ).

The structural peptoid isomers bearing three $\mathbf{H}$ and one $\mathbf{C}$ units have lifetimes ranging from 432-679 $\mu$ s (ESI, Table S $2 \dagger$ ) with the most noteworthy difference between (3) HCHH and (4) HHCH. By exchanging $\mathbf{C}$ units in the $2^{\text {nd }}$ and $3^{\text {rd }}$ positions we observe a ca. $250 \mu$ s change in $\mathrm{Eu}^{3+}$ lifetime, which corresponds to one and two bounds water molecules, respectively. ${ }^{34}$ This is indicative of a possible change in coordination of $\mathrm{Eu}(\mathrm{III})$ by (3) and (4) as a result of the difference in the order of $\mathbf{H}$ and $\mathbf{C}$ binding units, which suggests the location of $\mathbf{H}$ and $\mathbf{C}$ moieties may enhance/decrease recognition of $\mathrm{Eu}^{3+}$. The structural peptoid isomers bearing two $\mathbf{H}$ and two $\mathbf{C}$ units have lifetimes ranging from 425-553 $\mu$ s (ESI, Table S2 $\dagger$ ) and an analogous comparison of Eu:peptoid complexes where $\mathbf{C}$ units are exchanged in the $2^{\text {nd }}$ and $3^{\text {rd }}$ positions ((7) CCHH and (10) CHCH) yields a difference of only $c a .25 \mu \mathrm{s}$, which is a much smaller change than what was noted above. For peptoid isomers with two $\mathbf{H}$ and two $\mathbf{C}$ units the more interesting results come from comparing complexes with $\mathbf{H}$ units in the $3^{\text {rd }}$ and $4^{\text {th }}$ positions. We observe decreases of $c a$. 75-125 $\mu$ s in Eu(III) lifetimes when $\mathbf{H}$ moieties are switched from the $3^{\text {rd }}$ position to the $4^{\text {th }}$ position (comparing (5) CHHC with (10) CHCH and (8) HCHC with (9) HCCH), which is also possibly indicative of changes in ligand conformation about the Eu(III) metal center as a result of changing the order of binding moieties. The structural peptoid isomers bearing one $\mathbf{H}$ and three $\mathbf{C}$ units have lifetimes ranging from 569-682 $\mu$ s (ESI, Table S2 $\dagger$ ) and display the least amount of variance (in lifetime values) with changes in the order of the binding units, suggesting that once a ligand includes a certain number of $\mathbf{C}$ units (three) only one binding 
conformation, about the $\mathrm{Eu}^{3+}$ metal center, is observed. Noteworthy, Gd coordination properties will be investigated in the future for those four ligand structures with which $\mathrm{Eu}^{3+}$ luminescence lifetimes suggested two inner-sphere water molecules, (3) HCHH, (7) CCHH, (9) HCCH, and (10) CHCH. The ability to form Gd:peptoid complexes with q values of 2 may result in enhanced relaxivity properties, which could prove attractive for magnetic resonance imaging applications when associated with high thermodynamic stability constants, as detailed below.

\section{Solution thermodynamics}

Inspired by previous efforts of our group to characterize 3,4,3$\mathrm{LI}(\mathrm{CAM}),{ }^{14}$ we sought to investigate the solution thermodynamic properties of the peptoid analogue (15) CCCC. The last $3 \mathrm{p} K_{\mathrm{a}}{ }^{\prime} \mathrm{s}$ of (15) CCCC were not resolvable by spectrophotometric titrations and were held constant at values comparable to the spermine analogue. The proton-independent stability constant $\left(\log \beta_{110}\right)$ of the (15) CCCC complex with $\mathrm{Eu}^{3+}$ was refined to $28.88 \pm 3.45$ (ESI, Table $\mathrm{S} 3 \dagger$ ) and this result is quite similar to $\mathrm{Eu}^{3+}$ complexed with 3,4,3-LI(CAM) $\left(\log \beta_{110}=29.65\right) \cdot{ }^{14}$ Speciation modeling for equal concentrations of 3,4,3-LI(CAM) and (15) CCCC in the presence of $\mathrm{Eu}^{3+}$ reveals that 3,4,3-LI(CAM) predominately binds $\mathrm{Eu}^{3+}$ above a pH of 6 and further, the speciation diagram demonstrates that (15) CCCC is also fully binding $\mathrm{Eu}^{3+}$ under the conditions employed for fluorescence measurements (ESI $\dagger$ ). Incorporating a mixture of CAM and HOPO groups onto a scaffold was expected to increase the affinity of the chelator to the metal while increasing the $\mathrm{pH}$ range at which it binds, in comparison to the parent ligands. The stability constant $\left(\log \beta_{110}\right)$ of 3,4,3- $\mathrm{LI}(\mathrm{CHHC})$ complexed with $\mathrm{Eu}^{3+}$ is $35.97 \pm 0.06$ (ESI, Table S3†). Thermodynamic modeling (Fig. 6) of 3,4,3-LI(1,2-HOPO), 3,4,3-LI(CAM), and 3,4,3- $\mathrm{LI}(\mathrm{CHHC})$ in the presence of $\mathrm{Eu}^{3+}$ at equal concentrations revealed that the predominant complexing ligand above a pH of 6 is the mixed 343-LI species 3,4,3-LI(CHHC). At lower pH values, 3,4,3-LI(1,2-HOPO) dominated while a small fraction of

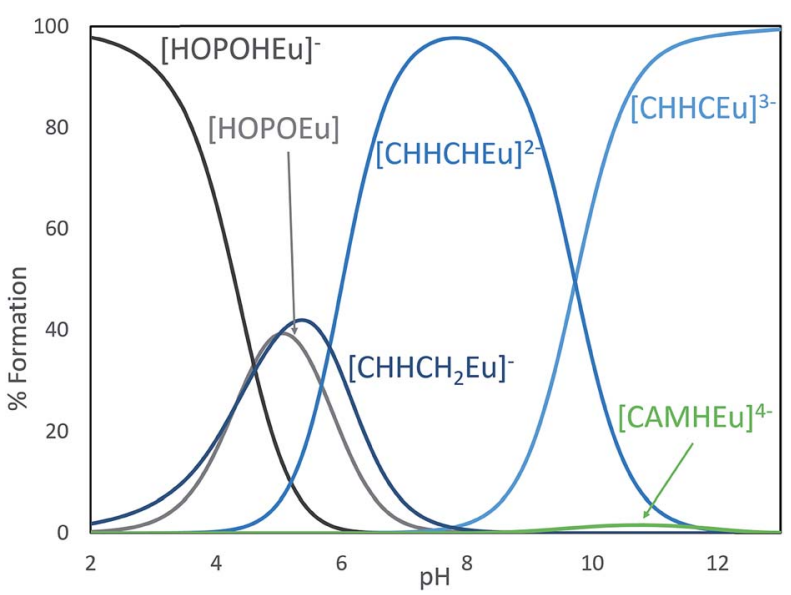

Fig. 6 Speciation diagram for a competition between 3,4,3-LI(1,2HOPO), 3,4,3-LI(CAM), and 3,4,3-LI(CHHC) abbreviated HOPO (gray), $\mathrm{CAM}$ (green), and $\mathrm{CHHC}$ (blue), respectively, in the presence of $\mathrm{Eu}^{3+}$. $[\mathrm{HOPO}]=[\mathrm{CAM}]=[\mathrm{CHHC}]=[$ metal $]=25 \mu \mathrm{M}, I=0.1 \mathrm{M}, \mathrm{T}=25^{\circ} \mathrm{C}$. Only the major species were labeled on the plots for clarity.
$3,4,3-\mathrm{LI}(\mathrm{CAM})$ is bound around $\mathrm{pH} 11$. The protonation constants for 3,4,3-LI(HCCH) were also investigated via spectrophotometric titrations, but the similarity of the Eu complex absorption signal with the signal from the free ligand $(3,4,3-\mathrm{LI}(\mathrm{HCCH}))$ prevented refinement with HypSpec. Therefore, competition batch titrations were employed against 3,4,3-LI(1,2-HOPO) (ESI $\dagger$ ). The $\log \beta_{111}$ value obtained for 3,4,3-LI(HCCH) was $43.34 \pm 0.49$. For comparison, we selected a peptoid with two $\mathbf{H}$ and two $\mathbf{C}$ moieties and the Eu complex formed with (7) $\mathbf{C C H H}$ displayed a stability constant $\left(\log \beta_{110}\right)$ of $36.01 \pm 4.13$, and is also fully formed at $\mathrm{pH}$ 7.4. As previously mentioned, having access to ligands with HOPO and CAM units opens up avenues for manipulating the properties of the chelator, as seen with the mixed ligands and when comparing the CAM analogues.

\section{Conclusions}

We report herein the synthesis of a new library of tetrameric peptoid ligands that can be used for chelation of f-block metals. We prepared and characterized a library of octadentate peptoids based upon 1,2-HOPO- and CAM-based sub-monomers, investigated their thermodynamic and photophysical properties with $\mathrm{Eu}^{3+}$ and $\mathrm{Tb}^{3+}$, and compared them with their spermine analogues. Both ligand systems show high affinity for Ln(III) metals with stability constants greater than $10^{29}$ for $\mathrm{Eu}^{3+}$ with the investigated chelators. Overall, the peptoid scaffold provides a platform to conduct a more comprehensive, systematic approach to study Ln-ligand interactions, and one can envision fine-tuning the peptoid manifold to incorporate the desired cavity size, binding moieties, and energetics of the chelator. As synthesis is done on solid-support, creativity and modularity can be imparted onto the ligand, depending on the desired function. We are now working on acquiring structural parameters of metal complexation, and further characterizing peptoid complexes with other f-block metals, by probing their ability to efficiently sensitize near-IR emitters as well as exploring the fundamental differences in energy transfer processes between $4 \mathrm{f}$ and $5 \mathrm{f}$ species. On-going work also focuses on expanding synthetic modifications of the scaffold for the development of targeted therapeutic applications.

\section{Materials and methods}

Chemicals were obtained from commercial suppliers and were used as received unless stated otherwise. The ligands 3,4,3-LI(1,2HOPO) and 3,4,3-LI(CAM) were prepared and characterized as previously described. ${ }^{14,35}$ Synthesis precursors 2,2-diphenylbenzo $[d][1,3]$ dioxole-4-carboxylic acid and 1,6-carboxy-1-(benzyl)hydroxy2-pyridinone were prepared according to literature procedures. ${ }^{14,36}$ While the mixed ligand 3,4,3-LI(CHHC) has been made before, ${ }^{7,37}$ herein we followed a slightly different approach and expanded efforts beyond what had been previously reported to produce both 3,4,3-LI(CHHC) and 3,4,3-LI(HCCH). Detailed syntheses and characterization of the two compounds can be found in the ESI. $\dagger$ All NMR spectra were recorded at ambient temperature on Bruker FT-NMR spectrometers, using tetramethylsilane as an internal reference. SilicaFlash G60 (particle size 60-200 $\mu \mathrm{m}$ ) was used for 
flash column chromatography. LC-MS was performed on an Agilent LC/MS system consisting of an Agilent 1200 binary LC pump, a temperature-controlled autosampler, a PDA UV detector, and a 6530 Accurate Mass Q-TOF mass spectrometer (Wilmington, DE, USA). The mass spectrometer was equipped with a JetStream ${ }^{\circledR}$ ESI probe operating at atmospheric pressure. The ESI source parameter settings were: mass range $m / z$ 100-1200, gas temperature $350{ }^{\circ} \mathrm{C}$, gas flow $10 \mathrm{~L} \mathrm{~min}{ }^{-1}$, nebulizer $50 \mathrm{psi}$, sheath gas temperature $400{ }^{\circ} \mathrm{C}$, sheath gas flow $12 \mathrm{~L} \mathrm{~min}^{-1}$, capillary voltage $\left(V_{\text {cap }}\right) 3500 \mathrm{~V}$, nozzle voltage $500 \mathrm{~V}$, fragmentor $200 \mathrm{~V}$, skimmer $65 \mathrm{~V}$, octopole RF (OCT 1 RF Vpp) $750 \mathrm{~V}$. Reverse phase preparatory HPLC was performed on a Varian ProStar system with a Vydac C18 column. HRMS and MS-MS were obtained on a Waters Xevo G2 Qtof mass spectrometer, and leucine encephalin lockspray with mass correction was used for HR-MS.

\section{Ethylenediamine substituted CAM submonomer, "C"}

2,2-Diphenylbenzo[ $d][1,3]$ dioxole-4-carboxylic acid $(3.84 \mathrm{~g}$, $12.06 \mathrm{mmol}$ ) was suspended in $30 \mathrm{ml}$ of toluene under an argon atmosphere. Oxalyl chloride $(1.14 \mathrm{ml}, 13.3 \mathrm{mmol})$ was then added followed by a catalytic amount of $\mathrm{N}, \mathrm{N}$-dimethylformamide. The suspension was heated to $40{ }^{\circ} \mathrm{C}$ and stirred until it became clear and the evolution of gas had ceased ( $\sim 1$ hour). The volatiles were then removed using the manifold vacuum and the resulting white solid was dissolved in dry $\mathrm{CH}_{2} \mathrm{Cl}_{2}$. A separate $1 \mathrm{~L}$ roundbottom flask outfitted with an addition funnel was charged with ethylenediamine ( $8 \mathrm{ml}, 120 \mathrm{mmol})$ and $50 \mathrm{ml}$ dry $\mathrm{CH}_{2} \mathrm{Cl}_{2}$; the resulting solution was cooled to $0{ }^{\circ} \mathrm{C}$ using an ice bath. The aforementioned solution of acyl chloride was transferred into the addition funnel and was diluted with $\mathrm{CH}_{2} \mathrm{Cl}_{2}$ to a total volume of $700 \mathrm{ml}$. The acyl chloride solution was then added into the vigorously stirred ethylenediamine over 1.5 hours at $0{ }^{\circ} \mathrm{C}$. Following the addition, the reaction solution was transferred into a separatory funnel and was washed with $0.5 \mathrm{M} \mathrm{NaOH}$ in $50 \%$ saturated aq. $\mathrm{NaCl}(50 \mathrm{ml} \times 2)$. The organic phase was dried over $\mathrm{MgSO}_{4}$ and was concentrated on a rotary evaporator yielding the crude. The crude was purified using silica column chromatography $\left(5 \rightarrow 10 \% \mathrm{MeOH}\right.$ in $\mathrm{CH}_{2} \mathrm{Cl}_{2}$ with $1 \% \mathrm{Et}_{3} \mathrm{~N}, R_{\mathrm{f}}=0.35$ in $10 \% \mathrm{MeOH}$ in $\mathrm{CH}_{2} \mathrm{Cl}_{2}$ ). The desired fractions were combined, concentrated under reduced pressure, and dried under vacuum yielding the CAM submonomer as a sticky yellow oil (3.49 g, $9.68 \mathrm{mmol}, 80 \%$ yield). ${ }^{1} \mathrm{H}$ NMR (300 $\left.\mathrm{MHz} \mathrm{CDCl}_{3}\right) \delta 7.64(1 \mathrm{H}$, br s, NH), 7.56-7.61 (5H, m, ArH), 7.377.42 (6H, m, ArH), 7.01 (1H, dd, $J=7.7,1.4 \mathrm{~Hz}, \mathrm{ArH}), 6.94(1 \mathrm{H}, \mathrm{t}$, $J=7.9 \mathrm{~Hz}, \mathrm{ArH}), 3.56\left(2 \mathrm{H}, \mathrm{q}, J=6.0 \mathrm{~Hz}, \mathrm{NHCH}_{2}\right), 2.97(2 \mathrm{H}, \mathrm{t}, J=$ $\left.6.0 \mathrm{~Hz}, \mathrm{NH}_{2} \mathrm{CH}_{2}\right), 2.75\left(2 \mathrm{H}, \mathrm{s}, \mathrm{NH}_{2}\right) .{ }^{13} \mathrm{C} \mathrm{NMR}\left(75 \mathrm{MHz}, \mathrm{CDCl}_{3}\right)$ $\delta$ 163.7, 147.2, 144.7, 139.4, 129.5, 128.4, 126.2, 122.4, 122.0, 118.0, 116.0, 111.6, 42.3, 41.5. HRMS-ESI $(\mathrm{m} / \mathrm{z})[\mathrm{M}+\mathrm{H}]$ calcd For $\mathrm{C}_{22} \mathrm{H}_{20} \mathrm{~N}_{2} \mathrm{O}_{3}+\mathrm{H}, 361.1563$; found, 361.1581.

\section{Ethylenediamine substituted 1,2-HOPO submonomer, "H"}

1,6-Carboxy-1-(benzyl)hydroxy-2-pyridinone ${ }^{36} \quad(3.84 \mathrm{~g}, \quad 12.06$ $\mathrm{mmol}$ ) was suspended in $30 \mathrm{ml}$ of toluene under an argon atmosphere. Oxalyl chloride $(1.14 \mathrm{ml}, 13.3 \mathrm{mmol})$ was then added followed by a catalytic amount of $N, N$-dimethylformamide. The suspension was heated to $40{ }^{\circ} \mathrm{C}$ and was stirred until it became clear and the evolution of gas has ceased ( $\sim 1$ hour). The volatiles were then removed using the manifold vacuum and the resulting white solid was dissolved in dry dichloromethane. A separate $1 \mathrm{~L}$ roundbottom flask outfitted with an addition funnel was charged with ethylenediamine $(8 \mathrm{ml}, 120$ $\mathrm{mmol}$ ) and $50 \mathrm{ml}$ dry dichloromethane; the resulting solution was cooled to $0{ }^{\circ} \mathrm{C}$ using an ice bath. The aforementioned solution of acyl chloride was transferred into the addition funnel and was diluted with dichloromethane to a total volume of $700 \mathrm{ml}$. The acyl chloride solution was then added into the vigorously stirred ethylenediamine over 1.5 hours at $0{ }^{\circ} \mathrm{C}$. Following the addition, the reaction solution was transferred into a separatory funnel and was washed with $0.5 \mathrm{M} \mathrm{NaOH}$ in $50 \%$ saturated aq. $\mathrm{NaCl}(50 \mathrm{ml} \times 2)$. The organic phase was dried over $\mathrm{MgSO}_{4}$ and was concentrated on a rotary evaporator yielding the crude. The crude was purified using silica column chromatography $\left(5 \rightarrow 10 \% \mathrm{MeOH}\right.$ in DCM with $1 \% \mathrm{Et}_{3} \mathrm{~N}, R_{\mathrm{f}}=$ 0.35 in $10 \% \mathrm{MeOH}$ in DCM). The desired fractions were combined, concentrated under reduced pressure, and dried under vacuum yielding the HOPO submonomer as a yellow oil (3.49 g, $9.68 \mathrm{mmol}, 80 \%$ yield). ${ }^{1} \mathrm{H}$ NMR (300 $\mathrm{MHz}, \mathrm{CDCl}_{3}$ ) $\delta$ 7.42-7.49 (3H, m, ArH and NH), 7.31-7.40 (3H, m, ArH), 7.26 $(1 \mathrm{H}, \mathrm{dd}, J=9.2,6.8 \mathrm{~Hz}, \mathrm{CHC} \underline{\mathrm{HCH}}), 6.67(1 \mathrm{H}, \mathrm{dd}, J=9.2,1.6 \mathrm{~Hz}$, $\mathrm{CHCHC} \underline{\mathrm{H}}), 6.44(1 \mathrm{H}, \mathrm{dd}, J=6.7,1.6 \mathrm{~Hz}, \mathrm{CHCHCH}), 5.28(2 \mathrm{H}, \mathrm{s}$, $\left.\mathrm{CH}_{2} \mathrm{Ph}\right), 3.36\left(2 \mathrm{H}, \mathrm{t}, J=6.0 \mathrm{~Hz}, \mathrm{NHCH}_{2}\right), 3.80(2 \mathrm{H}, \mathrm{q}, J=6.1 \mathrm{~Hz}$, $\left.\mathrm{NH}_{2} \mathrm{CH}_{2}\right) .{ }^{13} \mathrm{C} \mathrm{NMR}\left(75 \mathrm{MHz}, \mathrm{CDCl}_{3}\right) \delta 160.3,158.5,142.5,138.0$, 133.2, 130.1, 129.4, 128.6, 124.0, 106.4, 79.3, 42.7, 40.7. Calcd for $\mathrm{C}_{15} \mathrm{H}_{17} \mathrm{~N}_{3} \mathrm{O}_{3}+\mathrm{H}, 288.1328$; found, 288.1361.

\section{Synthesis of peptoids}

Unless noted otherwise, all steps were carried out in fritted polypropylene syringes, which allowed for recovery of submonomer for re-use. Automatic peptoid synthesis was not an option for this work due to difficulty of sub-monomer preparation. 100-150 mg of Rink amide resin was added to a fritted syringe. The resin was swollen by adding $2 \mathrm{ml}$ of DMF and rocking for 30 minutes, and then solution was ejected to isolate the swollen resin. $1 \mathrm{ml}$ of $20 \%$ 4-methylpiperidine in DMF (v/v) was added to deprotect the Fmoc group followed by agitation for two minutes, draining of the solution, and repeating for 12 minutes in total. Immediately following, the resin was rinsed with DMF $(2 \mathrm{ml}$, 5 times for 1 minute). To bromoacetylate, premix $0.8 \mathrm{M}$ bromo-

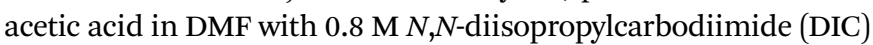
( $2 \mathrm{ml}$ total solution with $0.4 \mathrm{M}$ of each reagent). Draw the solution into the syringe, agitate for five minutes, and rinse again with $2 \mathrm{ml}$ DMF $(5 \times 1$ minute). In order for displacement to occur, draw in $1.5 \mathrm{ml}$ of submonomer solution (0.2 M in DMF), agitate for one1 hour at $45{ }^{\circ} \mathrm{C}$, and then rinse with DMF $(5 \times 1$ minute $)$. Repeat bromoacetylation and displacement steps until synthesis is finished and then wash with DCM $(3 \times 1$ minute $)$ after the last DMF wash. Finally, dry resin by pulling the plunger out and applying a gentle vacuum onto the syringe needle.

\section{Test cleavage}

A small amount of resin was placed into an LC-MS vial and was treated with $\sim 200 \mu \mathrm{l}$ of cleavage cocktail (95/2.5/2.5 TFA/water/ 
triisopropylsilane). The suspension was agitated for one hour at room temperature, after which it was diluted to $1 \mathrm{ml}$ with methanol and the beads were filtered off. The solution was analyzed by LC-MS for the desired mass. This procedure was only used analytically as benzyls from 1,2-HOPO containing peptoids are not cleaved under these conditions (keep this in mind when looking for the desired mass).

\section{Deprotection, cleavage, and purification}

Dry resin (100-150 mg) was placed into a scintillation vial and was swollen in $9 \mathrm{ml}$ of DCM by shaking for 30 minutes. $1 \mathrm{ml}$ of 1.0 $\mathrm{M} \mathrm{BBr}_{3}$ in hexanes was added via a syringe and the vial was capped and shaken for 60 minutes ensuring that all of the resin was thoroughly submerged, which removes benzyl protecting groups from HOPO units. The solvent was carefully removed with a glass pipette and the resin was washed with DCM $(2 \mathrm{ml})$ and methanol $(2 \mathrm{ml} \times 2)$, followed by DCM $(2 \mathrm{ml} \times 2)$. The peptoid was then cleaved from resin by treatment with cleavage cocktail for 60 minutes (the treatment also deprotects CAM units). The cleavage cocktail was filtered from resin and a small aliquot was removed and diluted with methanol for LC-MS analysis $\left(1 \rightarrow 30 \% \mathrm{MeCN}\right.$ in $\mathrm{H}_{2} \mathrm{O}$ over 20 minutes, both with $0.1 \%$ formic acid) while the resin was washed of TFA traces and discarded. Most LC-MS analyses showed a relatively clean desired compound. Iron complexes were sometimes observed, which we believe came from stainless steel components of the instrument. Volatiles were then removed from the filtrate using a vacuum pump. The resulting residue was dissolved in 90/10 acetic acid/water $(0.5-1 \mathrm{ml})$ and the resulting clear solution was stirred at $42{ }^{\circ} \mathrm{C}$ and treated with water in $0.5 \mathrm{ml}$ increments. The solution turned turbid upon addition of water and slowly clarified with continued stirring (5-15 min between additions). A total of $\sim 2.5 \mathrm{ml}$ of water was added, at which point the solution remained turbid even with prolonged stirring. The turbid solution was taken up into a syringe and injected onto reverse-phase prep-HPLC through a $0.45 \mu \mathrm{m}$ filter in no more than $2.0 \mathrm{ml}$ batches ( $\sim 2$ injections per peptoid).

\section{Photophysical measurements}

UV-visible absorption spectra were recorded on a Varian Cary 5G double-beam absorption spectrophotometer, using either $1.00 \mathrm{~cm}$ or $2.0 \mathrm{~mm}$ path length quartz cells. Each of the measured peptoid solutions was $45.4 \mu \mathrm{M}(100 \mu \mathrm{M}$ for 3,4,3-LI ligands) with 1.1 equivalents of metal $\left(\mathrm{M}=\mathrm{Eu}^{3+}\right.$ or $\left.\mathrm{Tb}^{3+}\right)$, to ensure the absence of free ligand, and buffered in $50 \mathrm{mM}$ HEPES at $\mathrm{pH}$ 7.4. Fluorescence and time-resolved measurements were acquired on a HORIBA Jobin Yvon IBH Fluorolog-3 spectrophotometer. The excitation source was a xenon flash lamp. A $400 \mathrm{~nm}$ long-pass filter was used when the second harmonic of the excitation beam overlapped with the emission spectra. For luminescence measurements, the ligands $(5.0 \mu \mathrm{M})$ were mixed with 0.9 equivalents of metal $\left(\mathrm{M}=\mathrm{Eu}^{3+}\right.$ or $\left.\mathrm{Tb}^{3+}\right)$ to ensure full metal complexation in $50 \mathrm{mM}$ HEPES buffer $(\mathrm{pH}$ 7.4), and excited at the absorption maximum for each of the complex. Quantum yield measurements were obtained using the optical dilution method with quinine sulfate as a reference.
${ }^{38}$ Samples of quinine sulfate at various absorbance (0.02-0.1) were prepared in $50 \mathrm{mM}$ sulfuric acid. Ln complexes (complexes $=\mathrm{Tb}: 3,4,3-\mathrm{LI}(\mathrm{CHHC})$ or Eu:HHCH (4)) at various absorbance $(0.02-1)$ were prepared in $0.1 \mathrm{M} \mathrm{KCl}$, buffered in $50 \mathrm{mM}$ HEPES at $\mathrm{pH}$ 7.4. All samples were background corrected for solvent auto fluorescence. The ligand luminescence leakage for $\mathrm{Tb}^{3+}$ spectra was background corrected with QTIPlot. At least five samples were used per trial for both quinine sulfate and metal complexes. The reported quantum yields and uncertainty were based off three independent trials. Additionally, the triplet-state energies of the ligands were determined by preparing $\mathrm{Gd}^{3+}$ complexes in situ (91\% EtOH, 4.5\% DMSO, and 4.5\% $\mathrm{H}_{2} \mathrm{O}$ ) and measuring the phosphorescence of the ligand under cryogenic conditions. The main peak in 3,4,3-LI(CAM), 3,4,3-LI(1,2HOPO), CCCC (15), and HHHH (16) phosphorescence spectra was modeled by two Gaussian curves each and further used to model the triplet-state for the mixed peptoid and spermine ligands. It was assumed that the triple-state character of the mixed ligands was a linear combination of the pure CAM and 1,2-HOPO ligands.

\section{Solution thermodynamics}

Absorbance spectra for spectrophotometric titrations were recorded on an Ocean Optics USB 4000 spectrophotometer (slit $50 \mathrm{~nm}$, grating 600 grooves per mm, blaze $400 \mathrm{~nm}$ ) equipped with a PX-3 pulsed xenon or HPX-2000 high-powered xenon light source. Spectral data were acquired with a $1 \mathrm{~cm}$ path length dip probe (Ocean Optics, Inc.). A microCombi Electrode (Metrohm) $\mathrm{H}^{+}$-response electrode was calibrated prior to each titration and used in conjunction with a Metrohm Titrando 907 to measure the $\mathrm{pH}$ of the experimental solutions. Absorption and $\mathrm{pH}$ measurements were taken after each incremental addition of carbonate-free 0.1 M KOH (prepared from Baker Dilut-It concentrate) by the Metrohm autoburet. The instrument set-up was completely automated by in-house titration software. All spectrophotometric titration samples were prepared under acidic conditions $(\sim \mathrm{pH} 1)$ with $25 \mu \mathrm{M}$ ligand buffered in HEPES, CHES, MES, and acetic acid ( $3 \mathrm{mM}$ each) in $0.1 \mathrm{M}$ ionic strength ( $\mathrm{KCl}$ supporting electrolyte) at $25^{\circ}$ under positive argon pressure. Solutions used for stability constants had 1 equivalent of metal $\left(\mathrm{M}=\mathrm{Eu}^{3+}\right)$. Data from each titration were imported and refined by the nonlinear least squares fitting program HypSpec (see ESI $\dagger$ ). The stability constant of Eu:3,4,3$\mathrm{LI}(\mathrm{HCCH})$ complex was determined by fluorimetric batch titrations. Solutions of $100 \mathrm{nM} \mathrm{Eu}: 3,4,3-\mathrm{LI}(\mathrm{HCCH})$, with varying equivalents of 3,4,3-LI(1,2-HOPO) $(\sim 0.6-32)$ buffered in $50 \mathrm{mM}$ HEPES at $\mathrm{pH} 7.4$ at $0.1 \mathrm{M}$ ionic strength ( $\mathrm{KCl}$ supporting electrolyte) were prepared, allowed to equilibrate at $25^{\circ}$ for at least 1 day (until no spectral change could be noted) and emission spectra recorded. The analytical signal used was from the Eu:3,4,3-LI(1,2-HOPO) complex, as previously described. ${ }^{24}$

\section{Author contributions}

IC, AR, and RJA designed the research. IC developed the synthetic pathway for peptoid ligands and IC, AR, and BN 
prepared all ligands. AR and KPC performed the spectroscopic and thermodynamic analyses of metal complexes. GJPD assisted with experimental design of solution thermodynamic measurements and interpretation of thermodynamic and spectroscopic data. All authors discussed the experimental results and contributed to the manuscript. All authors have given approval to the final version of the manuscript.

\section{Conflicts of interest}

IC and RJA are listed as inventors on a patent application filed by the Lawrence Berkeley National Laboratory and describing inventions related to the research results presented here. The authors declare no other competing financial interest.

\section{Acknowledgements}

Early peptoid library development was supported by the Fuel Cycle Research and Development Campaign (FCRD)/Fuel Resources Program, Office of Nuclear Energy, the U.S. Department of Energy (DOE) under Contract No. DE-AC02-05CH11231 at the Lawrence Berkeley National Laboratory (LBNL). Spermine-based ligand development and all spectroscopic characterization were supported by the DOE, Office of Science Early Career Research Program and Office of Science, Office of Basic Energy Sciences, Chemical Sciences, Geosciences, and Biosciences Division at LBNL under Contract DE-AC0205CH11231. Peptoid ligand purification and analysis were performed at the Molecular Foundry, a User Facility supported by the Office of Science, Office of Basic Energy Sciences, of the DOE under Contract No. DE-AC02-05CH11231. The authors thank Drs Ronald Zuckermann and Michael Connolly from the LBNL Molecular Foundry for invaluable help with peptoid preparation.

\section{References}

1 J.-C. G. Bünzli, Coord. Chem. Rev., 2015, 293-294, 19-47.

2 J.-C. G. Bünzli, in Handbook on the Physics and Chemistry of Rare Earths, ed. J.-C. G. Bünzli and V. K. Pecharsky, Elsevier, 2016, ch. 287, vol. 50, pp. 141-176.

3 C. S. Cutler, H. M. Hennkens, N. Sisay, S. Huclier-Markai and S. S. Jurisson, Chem. Rev., 2013, 113, 858-883.

4 R. D. Teo, J. Termini and H. B. Gray, J. Med. Chem., 2016, 59, 6012-6024.

5 A. J. Amoroso, I. A. Fallis and S. J. A. Pope, Coord. Chem. Rev., 2017, 340, 198-219.

6 J. A. Rees, G. J. P. Deblonde, D. D. An, C. Ansoborlo, S. S. Gauny and R. J. Abergel, Sci. Rep., 2018, 8, 4419.

7 A. E. V. Gorden, J. Xu, K. N. Raymond and P. Durbin, Chem. Rev., 2003, 103, 4207-4282.

8 L. S. Natrajan, A. N. Swinburne, M. B. Andrews, S. Randall and S. L. Heath, Coord. Chem. Rev., 2014, 266-267, 171-193.

9 G. J. P. Deblonde, M. Sturzbecher-Hoehne, P. B. Rupert, D. D. An, M.-C. Illy, C. Y. Ralston, J. Brabec, W. A. de Jong, R. K. Strong and R. J. Abergel, Nat. Chem., 2017, 9, 843-849.
10 G. J.-P. Deblonde, M. P. Kelley, J. Su, E. R. Batista, P. Yang, C. H. Booth and R. J. Abergel, Angew. Chem., Int. Ed., 2018, 57, 4521-4526.

11 K. P. Carter, R. G. Surbella III, M. Kalaj and C. L. Cahill, Chem.-Eur. J., 2018, 24, 12747-12756.

12 G. J. P. Deblonde, M. Sturzbecher-Hoehne and R. J. Abergel, Inorg. Chem., 2013, 52, 8805-8811.

13 M. Sturzbecher-Hoehne, T. A. Choi and R. J. Abergel, Inorg. Chem., 2015, 54, 3462-3468.

14 I. Captain, G. J. P. Deblonde, P. B. Rupert, D. D. An, M.-C. Illy, E. Rostan, C. Y. Ralston, R. K. Strong and R. J. Abergel, Inorg. Chem., 2016, 55, 11930-11936.

15 T. A. Pham, A. B. Altman, S. C. E. Stieber, C. H. Booth, S. A. Kozimor, W. W. Lukens, D. T. Olive, T. Tyliszczak, J. Wang, S. G. Minasian and K. N. Raymond, Inorg. Chem., 2016, 55, 9989-10002.

16 G. M. Figliozzi, R. Goldsmith, S. C. Ng, S. C. Banville and R. N. Zuckermann, in Methods in Enzymology, Academic Press, 1996, vol. 267, pp. 437-447.

17 A. S. Knight, E. Y. Zhou, J. G. Pelton and M. B. Francis, J. Am. Chem. Soc., 2013, 135, 17488-17493.

18 A. S. Knight, E. Y. Zhou and M. B. Francis, Chem. Sci., 2015, 6, 4042-4048.

19 M. Baskin and G. Maayan, Chem. Sci., 2016, 7, 2809-2820.

20 B. F. Parker, A. S. Knight, S. Vukovic, J. Arnold and M. B. Francis, Ind. Eng. Chem. Res., 2016, 55, 4187-4194.

21 A. S. Knight, R. U. Kulkarni, E. Y. Zhou, J. M. Franke, E. W. Miller and M. B. Francis, Chem. Commun., 2017, 53, 3477-3480.

22 M. Baskin and G. Maayan, Dalton Trans., 2018, 47, 1076710774.

23 M. Sturzbecher-Hoehne, C. Ng Pak Leung, A. D'Aleo, B. Kullgren, A.-L. Prigent, D. K. Shuh, K. N. Raymond and R. J. Abergel, Dalton Trans., 2011, 40, 8340-8346.

24 R. J. Abergel, A. D'Aléo, C. Ng Pak Leung, D. K. Shuh and K. N. Raymond, Inorg. Chem., 2009, 48, 10868-10870.

25 L. J. Daumann, D. S. Tatum, B. E. R. Snyder, C. Ni, G.-l. Law, E. I. Solomon and K. N. Raymond, J. Am. Chem. Soc., 2015, 137, 2816-2819.

26 W. T. Carnall, P. R. Fields and K. Rajnak, J. Chem. Phys., 1968, 49, 4424-4442.

27 K. Binnemans, Coord. Chem. Rev., 2015, 295, 1-45.

28 M. Latva, H. Takalo, V.-M. Mukkala, C. Matachescu, J. C. Rodríguez-Ubis and J. Kankare, J. Lumin., 1997, 75, 149-169.

29 V. V. Utochnikova and N. P. Kuzmina, Russ. J. Coord. Chem., 2016, 42, 679-694.

30 M. Congiu, M. Alamiry, O. Moudam, S. Ciorba, P. R. Richardson, L. Maron, A. C. Jones, B. S. Richards and N. Robertson, Dalton Trans., 2013, 42, 13537-13545.

31 X. Q. Song, Q. F. Zheng, L. Wang and W. S. Liu, Luminescence, 2012, 27, 459-465.

32 R. D. Archer, H. Chen and L. C. Thompson, Inorg. Chem., 1998, 37, 2089-2095.

33 S. Sato and M. Wada, Bull. Chem. Soc. Jpn., 1970, 43, 19551962. 
34 T. Kimura, R. Nagaishi, Y. Kato and Z. Yoshida, J. Alloys Compd., 2001, 323-324, 164-168.

35 R. J. Abergel, P. W. Durbin, B. Kullgren, S. N. Ebbe, J. Xu, P. Y. Chang, D. I. Bunin, E. A. Blakely, K. A. Bjornstad, C. J. Rosen, D. K. Shuh and K. N. Raymond, Health Phys., 2010, 99, 401-407.
36 M. A. Deri, S. Ponnala, P. Kozlowski, B. P. Burton-Pye, H. T. Cicek, C. Hu, J. S. Lewis and L. C. Francesconi, Bioconjugate Chem., 2015, 26, 2579-2591.

37 L. C. Uhlir, P. W. Durbin, N. Jeung and K. N. Raymond, J. Med. Chem., 1993, 36, 504-509.

38 C. Würth, M. Grabolle, J. Pauli, M. Spieles and U. ReschGenger, Nat. Protoc., 2013, 8, 1535. 